Relations industrielles

Industrial Relations

\title{
Initiation aux nouvelles techniques de gestion industrielle, par André Olmi, Entreprise Moderne d'Édition, Paris, 1967, 60 pp.
}

\section{Bertrand Belzile}

Volume 23, numéro 2, 1968

URI : https://id.erudit.org/iderudit/027913ar

DOI : https://doi.org/10.7202/027913ar

Aller au sommaire du numéro

Éditeur(s)

Département des relations industrielles de l'Université Laval

ISSN

0034-379X (imprimé)

1703-8138 (numérique)

Découvrir la revue

Citer ce compte rendu

Belzile, B. (1968). Compte rendu de [Initiation aux nouvelles techniques de gestion industrielle, par André Olmi, Entreprise Moderne d'Édition, Paris, 1967, 60 pp.] Relations industrielles / Industrial Relations, 23(2), 373-374.

https://doi.org/10.7202/027913ar

Tous droits réservés @ Département des relations industrielles de l'Université Laval, 1968
Ce document est protégé par la loi sur le droit d'auteur. L'utilisation des services d'Érudit (y compris la reproduction) est assujettie à sa politique d'utilisation que vous pouvez consulter en ligne.

https://apropos.erudit.org/fr/usagers/politique-dutilisation/ 
a l'autoritarisme des seconds et à la dose d'irrotionnalité qui l'accompagne parfois.

\section{Bernard SOLASSE}

\section{L'entretien d'appréciation, par Norman Maier,} Entreprise Moderne d'Edition, Paris, 1968, 290 poges.

L'entretien d'appréciation est abordé ici comme un excellent moyen de combler périodiquement l'impersonnalité des rapports supérieurs-subordonnés dans la grande entreprise moderne. L'entretien o d'autres fonctions considérées parfois plus importantes, telles apprécier le travail et les attitudes des subordonnés, corriger des positions troubles, régler des conflits particuliers. Puisque l'entretien doit prendre la forme d'un dialogue, il est une occasion particulièrement intéressante pour les deux personnes concernées de se juger mutuellement, de récrienter leur action sans qu'il soit nécessaire de respecter le niveau hiérarchique. Certains diront que l'appréciation sera plus efficace si l'entretien ne comporte aucune appréciation du personnel.

Normon Maier fait plus qu'exposer une nouvelle conception de base des entretiens d'appréciation, il donne des méthodes de dialogue. Ceux qui recherche les solutions toutes prêtes en trouveront. Six formes de dialogues sont présentées et onalysées systématiquement. Chaque mot, pourrait-on dire, est pesé, chaque attitude est étudiée non seulement en fonction des objectifs poursuivis par le supérieur, mais aussi en fonction des réactions et des besoins du subordonné. D'aucun comprendront qu'on ne peut composer à l'avance un dialogue qui se veut spontané et qui met surtout en présence des personnes qui se connaissent souvent très mal. D'ailleurs, chaque subordonné est un type particulier, non seulement par les situations différentes qu'il a connues, mais aussi par sa personnalité même, so façon de comprendre les choses et de réagir face à son supérieur. Attention donc à ces formules magiques!

Chacun des six dialogues commentés par Norman Maier peut servir beaucoup plus adéquatement d'instrument pour critiquer, commenter et analyser les dialogues que nous avons l'occasion de mener nous-mêmes. En effet, l'auteur dégage après chaque dialogue les grandes variables et l'exploitation qui en est faite. Ces variables pourront servir à tout le monde car elles sont les principes de base de tout entretien d'appré- ciation, par exemple lo participation, I'information, l'évaluation...

Le livre de Norman Maier portera davantage de fruits $s^{\prime} i l$ réussit simplement ò susciter de nouvelles mentalités et attitudes de la part des supérieurs à l'endroit des subordonnés, surtout à l'occasion de l'entretien périodique d'appréciation qui peut être soit une excellente occasion de nouer des ropports amicaux, soit une critique bête qui créera plus de tension qu'elle n'en déliera. Le succès de la méthode est dans l'esprit plus que dans les formules neuves.

\section{L.-René PARENTEAU}

Comment étudier un problème, par Roland Caude, Entreprise Moderne d'Edition, Poris, 1968, 117 pp.

Ce petit livre bien écrit évite le piège du racolage vulgaire en dépit de ce que pouvait laisser craindre son titre. Mais, à notre avis, le lecteur peu cultivé ou peu expérimenté auxquels les conseils donnés pourraient sans doute servir, se laissera rebuter par les termes abstraits, tels qu' a anolyse » et "synthèse ", sons compter les références à Descartes; en revanche, les a cadres d'entreprise " auxquels s'adresse particulièrement cet ouvrage, nous dit-on, auront l'impression de retrouver des vérités que leurs études ou leur expérience quotidienne ont rendues pour eux depuis longtemps évidentes.

\section{Geneviève SOLASSE}

\section{Initiation aux nouvelles techniques de ges- tion industrielle, par André Olmi, Entre- prise Moderne d'Edition, Poris, 1967, 60 pp.}

L'auteur se propose de faire un tour d'horizon des principaux problèmes industriels qui peuvent être abordés et résolus par les techniques économiques. II insiste sur la nécessité d'aborder ces problèmes ovec un esprit scientifique qui consiste à \& soutenir le raisonnement humain, plus ou moins intuitif et empirique, par un raisonement mathématique $»$. On a donc de plus en plus recours, dans la gestion économique des entreprises, à la recherche opérationnelle définie par plusieurs comme la théorie de la décision appliquée ou comme une méthode scientifique dont le but est de fournir au personnel exécutif des éléments quantitatifs pouvant servir de base aux décisions concernant les opérations qu'il dirige. 
L'auteur semble donc assimiler les techniques économiques aux techniques quantitatives utilisées par les économistes d'entreprise, quoiqu'il fasse peu appel aux modèles mothématiques dans sa première partie qui se rapporte à l'investissement dans l'entreprise. II n'en reste pas moins normal et même nécessaire de rapprocher ces deux types de techniques.

On trouve d'abord une introduction générale, courte mais excellente, sur la prise de décisions rotionnelles. L'auteur étudie l'investissement dans l'entreprise aux points de vue suivants: la politique d'investissement, la classification des investissements, le moment d'investir, le choix des investissements et le financement des investissements. Dans la deuxième portie, sous le titre Programmation industrielle, monsieur Olmi troite de l'crdonnancement, des problèmes de la production en série, de la programmation linéaire, des méthode P.E.R.T. et dérivées et de la programmation dynamique.

II s'agit, bien entendu, d'une initiation aux nourelles techniques de gestion industrielle.

\section{Bertrand BELZILE}

Management and Machiavelli, par Antony Jay, Musson Book Co., Toronto, 1967, $224 \mathrm{pp}$

Pour Antony Jay, l'entreprise moderne présente les principaux traits qui caractérisent une société politique. Une entreprise cherche à maintenir ou à agrandir so part du marché ; un empire défend l'intégrité de son territoire ou tend à l'agrandir par la colonisation. Une entreprise, tout comme un Etat, doit réaliser une certaine unité de pensée et d'action, intégrer les divers éléments qui la composent. Une entreprise, tout comme un état, doit réprimer des querelles intestines.

Cette similitude fropponte incite Antony Jay d̀ dresser une multitude de parallèles entre les stratégies mises au point, dans le passé, par les grands chefs politiques, militaires et religieux et celles déployées par les dirigeants de grandes entreprises, telles que Ford, General Motors, IBM, etc. Tout en retenant quelques principes de Machiovel, il emprunte surtout sa démarche, c'est-d̀-dire l'étude des problèmes actuels à la lumière de sa propre expérience et celle des outres qui ont affronté des situations similaires dons le passé. Cette approche permet da Jay d'élaborer des principes et des stratégies qui peuvent être extrêmement utiles ò nos chefs d'entreprise octuels.
Si, par exemple, le chef d'entreprise éprouve quelques difficultés à intégrer dans son sein des firmes concurrentes récemment acquises, il pourrait lire ovec profit le chopitre IV: From Boronies to Empires. Lo fusion York-Lancaster qui ouvre la dynastie des Tudors s'apparente à la fusion des firmes Hollerith and Powers Samas pour créer International Computers and Tabulators permettont ainsi à cette dernière de tenir le coup devant IBM

En manipulant les appropriations budgétaires, le président d'une grande entreprise peut prévenir ou réprimer les dissentions qui peuvent survenir entre les vices-présidents ou les chefs des grandes divisions. Sur ce point, le chapitre $\vee$ fournit de nombreux conseils au président-roi qui lui serviront de guides dans ses relations ovec ses vicesprésidents-barons.

Un degré acceptable de décentralisation se trouve dans la manière dont les Etats ont fondé des colonies. Athènes et Rome dans I'antiquité; Venise et Gènes au Moyen Age; I'Espagne et l'Angleterre après la Renaissance ont connu beaucoup de succès avec leurs politiques de colonisation. Un directeur des ventes peut s'inspirer de ces enseignements pour constituer un groupe autonome de vendeurs chargés d'écouler un produit en territoire non-conquis

En délaissant momentonément l'histoire et la théorie politique, Jay réfère ò sa propre expérience pour présenter une critique constructive de la structure pyramidale de l'autorité dans l'entreprise. La structure de l'outorité édifiée selon les principes classiques en administration (the fearful symetry) peut conduire à un étouffement de l'initiative personnelle et de la créativité. En partant d'une notion de la créativité tirée du volume d'Arthur Koestler: "The Act of Creation a et il essaie d'esquisser un profil du dirigeant inventif et propose une refonte des structures ; il décrit ensuite le processus de la créativité, le genre de formation qu'il faudrait donner à nos gradués universitaires, la manière de constituer dans l'entreprise des groupes d'individus qui puissent produire et réaliser des idées nouvelles. II s'attaque au problème de détecter les vrais leciders capables de succéder avec brio aux dirigeants actuels. Richard Coeur de Lion, King Lear, Nicholas II, George I, George III et Edward le Confesseur sont autant d'exemples de maurvais leaders qui habitent la chambre des horreurs (The Chamber of Horrors, ch. XVII).

Une entreprise, comme un Etat, a besoin d'une croyance. Chaque membre est appelé 